\title{
Research on the Relationship between Newcomers' Self-socialization and Role Performance
}

\author{
Chih Huang
}

No.6, Jinfeng Rd., Tangjiawan, Zhuhai, Guangdong, China

\section{7@qq.com}

\begin{abstract}
Keywords: Organizational Socialization, Information-seeking Behaviors, Role Performance, Role Breadth Self-efficacy
\end{abstract}

\begin{abstract}
This study aims to explore the socialization issues of new-coming professional engineers. The purpose of this study is to investigate the relationship between information-seeking behaviors and role performance. Meanwhile, this study introduces role breadth self-efficacy to examine the mediating influence on information seeking behaviors-role performance relationship. Collecting data from 91 groups, which include 91 immediate supervisors and 384 new-coming professional engineers, the results show that (1) when individuals apply overt or observation as the information-seeking behavior, there is a positive effect on role performance respectively, (2) role breath self-efficacy has full mediating effect on the positive relationship between overt, observation and role performance individually.
\end{abstract}

\section{新进人员自我社会化与角色绩效的关系研究}

\author{
黃智 \\ 北京理工大学珠海学院, 广东省珠海市, 中国 \\ 2753426367@qq.com
}

关键词：组织社会化；信息寻找行为；角色绩效；角色宽度自我效能

中文摘要.本研究以新进专业工程人员社会化的问题为核心，探讨信息寻找行为对角色绩效之 影响, 并引入角色宽度自我效能作为中介变量, 以弥补以往的研究缺口。本研究设计采主管 和部属配对方式进行测量(主管91名，员工384名), 研究结果显示，新进人员的直接询问和观 察的信息寻找行为分别对角色绩效有显着正向影响，而角色宽度自我效能中介了直接询问和 观察信息寻找行为与角色绩效的正向关系。

\section{1. 引言}

组织社会化对组织运营和新进员工的工作调适都是相当重要的过程(Jokisaari and Nurmi, 2009)。尤其, 组织并无法提供新进人员所有的社会化信息, 因此, 员工个人必须通过透过信 息寻找行为进行自我社会化(Finkelstein, Kulas, \& Dages, 2003), 以获得工作所需的知识和技能, 提升对新工作环境的了解、预测及控制能力。

本研究尝试从探讨新进人员信息寻找行为与角色绩效的关系，以改善过去的研究探讨新 进人员社会化与工作调适关系时缺少对主要基准的连结(例如:工作绩效)。其次，以往的研究 较缺乏中介效应的探讨，而可能导致对新进人员社会化过程的理解有所限制(Ashforth, Sluss, and Saks, 2007)。因此, 本研究加入角色宽度自我效能做为中介机制, 以提供新进人员在工作 角色学习的改变和调适过程提供较为明确的解释。 


\section{2. 理论背景与假设}

组织社会化是新进人员刚进入组织时，获得有关工作角色及适应工作情境的知识、技能 的过程。然而, 受到组织、主管或同事并无法提供完整的社会化信息的限制, 因此, 新进人 员主动的信息寻找行为就成为获得承担工作角色所需知识与技能和因应工作环境的有效策略。

从过去众多的相关实证研究的回顾发现, 新进人员信息寻找行为以直接询问和使用频率 高于其他信息寻找行为(e.g., Morrison, 1993)。Jablin (2001)指出当新进人员针对想要了解的信 息以直接向主管或同事询问, 较能有效获得明确的信息, 对承担工作角色的认知较能赋予正 向与合理的意义。近年来, 众多组织对内部跨部门的功能整合相当频繁, 强调员工需更主动 及愿意执行较为宽广的工作角色。Welbourne, Johnson, \& Erez (1998)指出工作绩效不仅于特定 工作角色的技能表现, 其工作角色外的行为对团队、组织整体绩效亦有相当程度的影响。本 研究预期新进人员的信息寻找行为对角色绩效有所影响。在直接询问的信息寻找行为方面, 新进人员若能直接询问问题时, 主管或同事能适地给予厘清或导正, 对其工作问题提供适时 的协助, 将有助于新进人员绩效的提升。在观察的信息寻找行为方面, 新进人员通过观察主 管或同事的行为, 可掌握完成工作成功与失败的线索, 并获得工作行为的指导准则, 应有助 于提升对其执行多元工作角色的表现。基于以上的论述, 本研究提出以下待验证的假设:

假设 1：新进人员的信息寻找行为对角色绩效有显着的正向影响。

假设 1-1：新进人员的直接询问信息寻找行为对角色绩效有显着的正向影响。

假设 1-2: 新进人员的观察信息寻找行为对角色绩效有显着的正向影响。

角色宽度自我效能是员工对自身执行多元工作组合之能力的判断(Parker，1998)，其与自 我效能都强调个体对能完成工作的认知和评估。Torkzadeh \& Van Dyke (2001)指出, 自我效能 具有延展性, 个人会因获得新的信息、经验或回馈而产生改变。Luszczynska, Scholz \& Schwarzer (2005) 则认为个人若具有高自我效能不仅会改进目标设定, 对目标追求也较为坚持 不解; 同时, Luszczynska 等人也指出自我效能是预测行为的重要中介因子, 其经由影响个人 的目标意图而间接地影响行为。据此, 本研究提出以下待验证的假设:

假设 2：角色宽度自我效能会中介信息寻找行为与角色绩效的正向关系。

假设 2-1：角色宽度自我效能会中介直接询问信息寻找行为与角色绩效的正向关系。

假设 2-2：角色宽度自我效能会中介观察信息寻找行为与角色绩效的正向关系。

\section{3. 研究方法}

\section{1 样本与程序}

本研究以台湾高科技产业, 包括生化科技、光电、积体电路、通讯产业、精密机械、计 算机及外围等工程专业部门或单位的主管及新进全职之专业工程人员, 并将「新进人员」界 定在进入公司在 3-12 个月内的全职专业工程员工, 以符合社会化理论对新进人员的定义 (Bauer, Morrison, \& Callister, 1998)。其次, 本研究的研究设计是采主管与直属员工的配对方 式进行测量, 每一工作团队主管人员 1 名及直属新进员工至少 3-5 名, 共发出 960 份问卷 (主管 160 份问卷, 员工 800 份), 回收后经扣除无法配对、填答不完整及资格条件不符者后, 有效问卷为 475 份，共计 91 个群组(主管 91 份，员工 384 份), 配对成功率为 $49.46 \%$ 。

\section{2 測量}

在变量的测量方面, 信息寻找行为量表引用 Miller(1996)所编制的量表之「直接询问」及 「观察」两个维度进行衡量, Cronbach' $\mathrm{s} \alpha$ 值分别为 .83 及.72。角色宽度自我效能的测量 则采用 Parker(1998)所发展的量表进行测量, 为单一维度, Cronbach's $\mathrm{s} \alpha$ 值为 .90。角色绩效 的测量采用 Welbourne 等学者(1998)所发展之量表, 为单一维度, Cronbach's $\mathrm{s} \alpha$ 值为 .90。 另外, 本研究引入进入组织时间长短、工作经验、正向情感、负向情感等做为控制变量。 


\section{4. 研究结果}

表 1 的复回归分析结果显示，模型 2 中的直接询问及观察的信息寻找行为对角色绩效有 显着的正向影响, 因此,假设 1-1 及假设 1-2 获得支持。接着, 本研究依据 Baron \& Kenny (1986) 的中介影响确认条件，依序对角色宽度自我效能的中介条件是否成立进行检视。如表 1 中的 模型 5 所示, 直接询问和观察的回归系数皆达显着水准, 显示直接询问和观察的信息寻找行 为分别对角色宽度自我效能具有显着的正向影响。其次, 表 1 中的模型 4 可知, 角色宽度自 我效能的回归系数达显着水准，显示角色宽度自我效能对角色绩效有显着的正向影响。经确 认前述验证中介影响的先决条件成立后, 进行角色宽度自我效能在信息寻找行为与角色绩效 关系之间的中介效果验证。由表 1 中的模型 2 与模型 3 比较, 发现当模型 3 引入角色宽度自 我效能之变量后，直接询问和观察的信息寻找行为之回归系数均由显着变为不显着。

表 1 角色宽度自我效能对信息寻找和角色绩效的阶层回归分析

\begin{tabular}{|c|c|c|c|c|c|c|}
\hline \multirow[b]{2}{*}{ 自变量 $\downarrow$} & \multirow[t]{2}{*}{ 因变量 $\rightarrow$} & \multicolumn{4}{|c|}{ 角色绩效 } & \multirow{2}{*}{$\begin{array}{c}\text { 角色宽度自我 } \\
\text { 效能 } \\
\text { 模型 } 5\end{array}$} \\
\hline & & 模型 1 & 模型 2 & 模型 3 & 模型 4 & \\
\hline 常数项 & & $2.50^{* * * *}$ & $1.30^{* *}$ & $.98^{* *}$ & $1.90^{* * * *}$ & $.81^{*}$ \\
\hline 进入组织时间长短 & & .18 & .16 & .16 & & -.01 \\
\hline 工作经验 & & .02 & .01 & .02 & & -.00 \\
\hline 正向情感 & & $.16^{* *}$ & .08 & .04 & & $.10^{*}$ \\
\hline 负向情感 & & .06 & .05 & .04 & & .03 \\
\hline 直接 & & & $.22^{* *}$ & .10 & & $.31^{* * *}$ \\
\hline 观察 & & & $.21^{* * *}$ & .10 & & $.27^{* * *}$ \\
\hline 角色宽度自我效能 & & & & $.39^{* * *}$ & $.45^{* * *}$ & \\
\hline$F$ & & $3.07^{* * *}$ & $5.55^{* * *}$ & $8.52^{* * *}$ & $70.07^{\text {**** }}$ & $11.94^{* * * *}$ \\
\hline$R^{2}$ & & .02 & .07 & .16 & .15 & .15 \\
\hline
\end{tabular}

为进一步确认角色宽度自我效能的间接效应是否显着, 本研究以 sobel 检定进行检视, 检 定结果显示, 角色宽度自我效能分别在直接询问和观察的信息寻找行为与角色绩效的关系间 有显着的间接效应 $(\mathrm{z}>1.96, \mathrm{p}<.001)$ 。因此, 可以确认角色宽度自我效能在直接询问和观察的 信息寻找行为与角色绩效的关系间分别有完全中介的影响，假设 2-1, 2-2 获得支持。

\section{5. 结论与管理意涵}

本研究的结果显示, 直接询问和观察的信息寻找行为对新进人员执行多重工作任务的绩 效表现有正向影响, 因此, 部门或单位若能提供双向沟通管道及同侪友善的社会支持网络, 将有利于提升新进专业工程人员建立成功的工作经验及绩效表现。其次, 组织可实施师徒制, 新进人员通过对有经验且资深人员的观察, 产生模仿的学习移转效果, 缩短尝试错误和自我 摸索的时间, 对其工作及态度认知调整及后续的工作角色表现应有所帮助。另外, 员工的自 我效能可经由组织或工作群体的干预措施而提升, 故部门可藉由各种教育训练方案, 以提升 员工完成范围较广泛的工作角色的信心提升和绩效表现。本研究通过对新进人员社会化与工 作调适主要基准的连结(角色绩效), 并加入角色宽度自我效能的中介效应实证结果, 相较于 以往的研究, 对新进人员学习的转变和调适提供了更细致、完整过程而具贡献。 


\section{References}

[1] M, Jokisaari, and J. Nurmi, Change in newcomers' supervisor support and socialization outcomes after organizational entry, Academy of Management Journal, vol. 52, pp. 527-544, 2009.

[2] L. M. Finkelstein, J. T. Kulas, and K. D. Dages, Age differences in proactive newcomer socialization strategies in two populations, Journal of Business and Psychology, vol. 17, pp. 473-502, 2003.

[3] B. E. Ashforth, D. M. Sluss, and A. M. Saks, Socialization tactics, proactive behavior, and newcomer learning: Integrating socialization models, Journal of Vocational Behavior, vol. 70, 447-462, 2007.

[4] E. W. Morrison, Newcomer information seeking: Exploring types, modes, sources, and outcomes, Academy of Management Journal,, vol.36, pp. 557-589, 1993.

[5] F. M. Jablin, Organizational Entry, Assimilation, and Disengagement/Exit. In F. M. Jablin \& L. L. Puttnam (Eds), The New Handbook of Organizational Communication: Advances in Theory, Research, and Methods, pp. 732-818. Thousand Islands, CA: Sage, 2001.

[6] T. M. Welbourne, D. E. Johnson, and A. Erez, The role-based performance scale: Validity of a theory-based measure. Academy of Management Journal, vol.5, pp. 540-555, 1998.

[7] S. K. Parker, Role breadth self-efficacy: Relationship with work enrichment and other organizational practices, Journal of Applied Psychology, vol. 83, pp. 835-852, 1998.

[8] G. Torkzadeh, and T. P. Van Dyke, Development and validation of an internet self-efficacy scale, Behavior \& Information Technology, vol. 20, pp. 275-280, 2001.

[9] A. Luszczynzka, U. Scholz, and R. Schwarzer, The General self-efficacy scale: Multicultural validation studies. The Journal of Psychology, vol. 139, pp. 439-457, 2005.

[10]R. M. Baron, and D. A. Kenny, The Moderator-mediator variable distinction in social psychological research: conceptual, strategic, and statistical considerations, Journal of Personality and Social Psychology, vol. 51, pp. 1173-1182, 1986. 